\title{
A Matching Pursuit-Based Vehicle Wheel Parameter Extraction Method from Micro-Doppler Radar Signal
}

\author{
Lamei ZHANG, Yifan CHEN, Shuo LIU, Bin ZOU \\ Dept. of Information Engineering, Harbin Institute of Technology, Harbin 150001, China \\ lmzhang@hit.edu.cn, yifan_chen111@163.com, liushuo_1993@qq.com,zoubin@hit.edu.cn \\ Submitted July 6, 2021 / Accepted November 4, 2021
}

\begin{abstract}
Micro-Doppler effects of moving vehicles in a radar system are mainly induced by the rotation of wheels, whose features are closely related to the numbers, positions and radiuses of wheels. These parameters of wheels are critical for the vehicle classification and recognition. However, most micro-Doppler features extraction works of vehicles are unable to explicitly extract parameters of wheels. In this paper, a parameter extraction method of vehicle wheels using micro-Doppler features based on the matching pursuit (MP) is proposed. The micro-Doppler signals of wheels are generally weak comparing to redundant echo signals induced by other irrelevant parts of the vehicle, which makes the micro-Doppler features difficult to extract. In this case, several signal atom sets are created according to the motion states of irrelevant parts of vehicle and MP is performed to suppress the redundant signals. After the suppression, micro-Doppler signals induced by wheels have become the major part of the echo signal. Another atom set is generated according to the rotational motion of wheels to perform MP again. Then the wheel parameters, such as the estimated numbers, positions and radiuses, are extracted. Simulation results demonstrate that the proposed method is feasible in feature extraction of moving vehicle. Besides, the accuracy can be guaranteed when the signal-to-noise ratio is greater than $-5 d B$.
\end{abstract}

\section{Keywords}

Micro-Doppler, moving vehicle, feature extraction, matching pursuit (MP), radar echo analysis

\section{Introduction}

Recognition of vehicle via radar echo becomes increasingly important with the improvement of resolution and anti-jamming capability of the radar system. However, it remains challenging to discriminate the vehicles with fine distinctions, because their echo signals could be quite similar. The micro-Doppler effects are generated by the micro motion dynamics of small widgets in addition to the translational motion of target, such as the rotation of vehi- cle wheels and helicopter blades, the vibration of antennas, the motions of human legs, etc. [1-3]. Therefore, the micro-Doppler signatures contain the characteristics of the rotational vehicle wheels and have been proved to be critical for the vehicle's recognition with similar structures [4].

In order to observe the micro-Doppler effect and extract effective features, the echo signals first need to be preprocessed [5-7]. The simplest approaches are timefrequency analysis methods, including short-time Fourier transform (STFT), Wigner-Ville distribution (WVD), etc. STFT uses narrow window functions to perform piecewise Fourier transform, which is simple and easy to implement. Nevertheless, reaching high resolution simultaneously in time domain and frequency domain is extremely challenging [8]. WVD improves the resolution in both domains but the cross interference terms are introduced [9]. Signal decomposition and reconstruction is another commonly used technique for micro-Doppler signal preprocessing [10]. Empirical Mode Decomposition (EMD) is a commonly used method, which decomposes the signal into a series of Intrinsic Mode Functions (IMFs) [11]. Signal components of different frequencies can be reconstructed by this method. The matching pursuit (MP) has been proved to be another effective signal decomposition method. It decomposes arbitrary signal into the combination of basic waveforms in the dictionary, which is well suited to reconstruct different types of micro-Doppler signals [12-14]. After completion of the signal preprocessing, micro-Doppler features can be extracted further.

There has been a considerable number of studies on the micro-Doppler feature extraction. Nevertheless, few attempts have been made to directly extract the physical characteristics of targets. Based on the time-frequency spectrogram obtained by STFT, many micro-Doppler features can be extracted for target recognition or classification. In [15], features like the bandwidth of the targets' Doppler modulation are extracted from the spectrogram. Similar features are extracted in [16]. According to the spectrum processed by compressed sensing, the mean value, variance and entropy of the Doppler spectrum are extracted for further classification of targets. But the features are not related to the physical properties of the target. The micro-Doppler frequencies of human lower leg are 
estimated from the spectrogram in [17] to locate the moving human target. A new signal decomposition method, called short-time variational mode decomposition, is proposed in [18] to analyze the irregular micro-Doppler signals and estimate the instantaneous frequency of target. In [19], EMD is used to decompose the echo signal of the moving vehicle and six statistical features, such as energy ratio and amplitude ratio, are extracted from the IMFs. Several similar micro-Doppler features of moving vehicles are extracted from IMFs in [20]. In addition to statistical features, some geometrical features, like distance between two frequency peaks, are extracted as well. In [21], some fractal dimensions of micro-Doppler are extracted from the IMFs for classifying ground targets. The approaches of feature extraction are varied but these features cannot accurately describe the physical characteristics of targets.

According to the Doppler effect, the echo of a moving vehicle is a linear frequency modulated signal. Besides, an additional modulation effect induced by the rotation of wheels will be superimposed to the returned radar signal, by which the parameters of wheels can be extracted. Nevertheless, the signal components induced by rotational wheels are usually weak compared with those generated by the body of vehicle and the static scenes. Therefore, the sinusoid components are intricate and easily covered by other signal components, which make it intractable to extract micro-Doppler features from the echoes [22].

In this paper, a vehicle wheel parameter extraction method based on MP from micro-Doppler radar signal is proposed, which is applicable for vehicles with caterpillar band. Physical characteristics of wheels can be directly extracted from the micro-Doppler signal components, which are highly advantageous features in vehicle recognition and classification. But they are seldom paid attention to in recent studies about micro-Doppler feature extraction. According to our research, the micro-Doppler signal components are quite weak compared with other signals. Hence, the atom sets corresponding to the signals of static scene, the bodywork and the caterpillar band are created first to suppress those irrelevant signal components. After this, the rotational micro-Doppler signals will become the major components of the echo signal. An atom set corresponding to the rotational points is created to extract the micro-Doppler features of wheels, from which the vehicle wheel parameters, such as the estimated numbers, positions and radiuses, can be obtained. Parts of this study have been described briefly in our previous work [23]. However, the previous method is not able to guarantee the accuracy of estimated parameters for vehicle with caterpillar band. In this paper, the micro-Doppler signal components of caterpillar band are taken into consideration. More detailed analysis of micro-Doppler effects is given and the decision rule of the output estimated parameters is improved to get higher accuracy of parameter extraction. Moreover, the anti-noise capability of the algorithm is tested under different signal-to-noise ratios (SNRs).

The remaining paper is organized as follows. In Sec. 2, the signal model of a moving vehicle with caterpil- lar band is presented. The micro-Doppler effects of scattering points with different motion states are analyzed theoretically. In Sec. 3, the algorithm of irrelevant signal removal and wheel parameter extraction based on MP is described in details. At last, parameter extraction experiments of an armored car and a caterpillar tank are given in Sec. 4. The anti-noise capability of the algorithm is also analyzed. Concluding remarks are provided in Sec. 5 .

\section{Signal Model and Micro-Doppler Effect Analysis}

\subsection{Basic Concept of Micro-Doppler Effect}

The relative movement between the target and radar will cause a Doppler shift. Besides the Doppler frequency caused by the translational motion of a target, the micromotions of the scattering points will cause additional modulations to the returned signal of radar, which is called micro-Doppler effect. The micro-Doppler frequency can be obtained by means of baseband transformation and timefrequency analysis. Values of micro-Doppler frequencies are determined by the forms of the transmitted signal of radar and the instantaneous distances between the scattering points and radar. Therefore, to obtain the forms of micro-Doppler frequencies, the distances between radar and different types of scattering points need to be analyzed first.

\subsection{Mathematical Model of a Moving Vehicle}

A model of a tank with caterpillar band on the ground, shown in Fig. 1, is used to illustrate the variations of distances between radar and different types of scattering points. There are several kinds of scattering points with different motion states in the simulation scene. We analyze the micro-Doppler features of all these scattering points, which ensures that the parameter extraction method is applicable to most kinds of vehicles, including regular vehicles without caterpillar band.

All the simulation models in this paper are built by Google Sketchup. In order to analyze the instantaneous distances between radar and the scattering points with different motion states, the model is simplified into the form shown in Fig. 2. The radar is directional, whose line of sight is perpendicular to the moving direction of vehicle. The coordinate of the radar is $\left(0, H, R_{0}\right)$, where $H$ is the height of radar and $R_{0}$ is the vertical distance between radar and xoy plane.

Four kinds of scattering points of different motion states are analyzed in the simulation. Scattering point existing on the stationary object is represented as $N_{p}$, and $K_{n}$ is a translational scattering point existing on the main body of the vehicle. Scattering points on the wheels and caterpillar band are represented as $M_{q}$ and $L_{m}$ respectively. 


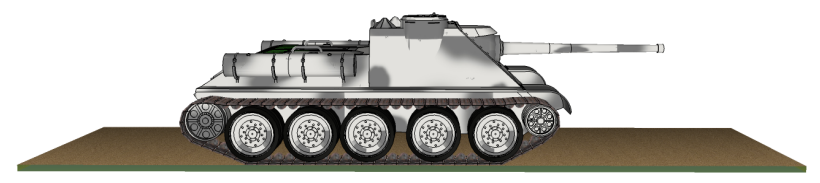

Fig. 1. Model of a tank with caterpillar band.

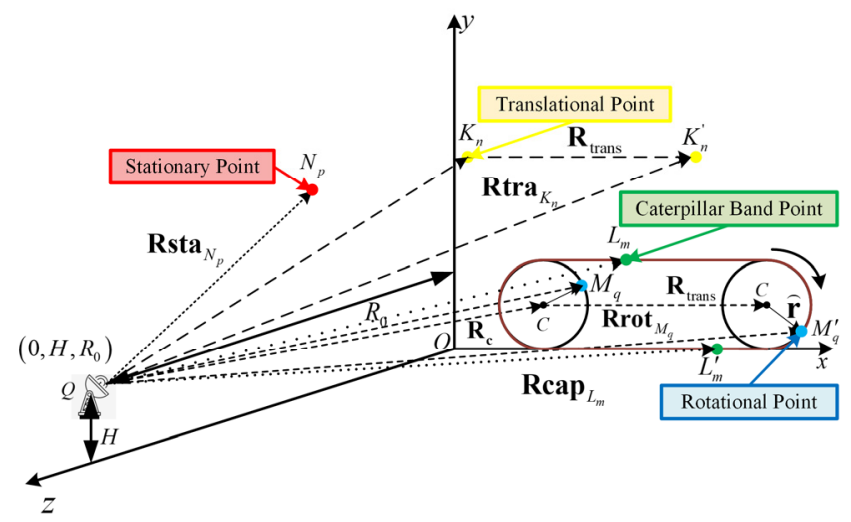

Fig. 2. Geometric model of a moving vehicle.

For the scattering point $N_{p}$ existing on the stationary objects, the distance between $N_{p}$ and the radar located at $Q$ is

$$
\begin{aligned}
R_{N_{p}} & =\left\|\operatorname{Rsta}_{N_{p}}\right\|=f\left(x_{N_{p}}, y_{N_{p}}, z_{N_{p}}\right) \\
& =\sqrt{x_{N_{p}}^{2}+\left(y_{N_{p}}-H\right)^{2}+\left(z_{N_{p}}-R_{0}\right)^{2}}
\end{aligned}
$$

where Rsta $N_{p}$ is the range vector from radar to the stationary point, $\left(x_{N_{p}}, y_{N_{p}}, z_{N_{p}}\right)$ is the coordinate of $N_{p}$ and \|\| is the Euclidean norm.

Assuming that the vehicle is moving along the $x$-axis in the positive direction with a velocity of $v$ the instantaneous distance between $K_{n}$ and radar is

$$
\begin{aligned}
R_{K_{n}}(t) & =\left\|\mathbf{R t r a}_{K_{n}}\right\|=\left\|\mathbf{Q} \mathbf{K}_{n}+\mathbf{R}_{\text {trans }}\right\| \\
& =f\left(x_{K_{n}}, y_{K_{n}}, z_{K_{n}}, v, t\right) \\
& =\sqrt{\left(x_{K_{n}}+v t\right)^{2}+\left(y_{K_{n}}-H\right)^{2}+\left(z_{K_{n}}-R_{0}\right)^{2}}
\end{aligned}
$$

where $\mathbf{R t r a}_{K_{n}}$ is the range vector from radar to the real time location of the translation point, $\mathbf{Q} \mathbf{K}_{n}$ is the range vector from radar to the initial location of the translation point and $\mathbf{R}_{\text {trans }}$ is the vector describing the translational motion. The initial coordinate of $K_{n}$ is $\left(x_{K_{n}}, y_{K_{n}}, z_{K_{n}}\right)$.

For the rotational scattering point $M_{q}$ on the wheels, its angular velocity is relevant to the radius of the wheel. The instantaneous distance between the radar and $M_{q}$ is

$$
\begin{aligned}
& R_{M_{q}}(t)=\left\|\operatorname{Rrot}_{M_{\mathrm{q}}}\right\| \\
& =\left\|\mathbf{R}_{\mathbf{c}}+\mathbf{R}_{\text {trans }}+\hat{\mathbf{r}}\right\|=f\left(x_{P_{c}}, y_{P_{c}}, z_{P_{c}}, \mathbf{v}, \widehat{\mathbf{r}}, t\right) \\
& =\left[\left(x_{P_{c}}+v t\right)^{2}+\left(y_{P_{c}}-H\right)^{2}+\left(z_{P_{c}}-R_{0}\right)^{2}+R_{\mathrm{r}}^{2}+\right. \\
& \left.2\left(x_{P_{c}}+v t\right) R_{\mathrm{r}} \cos (\omega t+\varphi)+2\left(y_{P_{c}}-H\right) R_{\mathrm{r}} \sin (\omega t+\varphi)\right]^{\frac{1}{2}}
\end{aligned}
$$

where $\mathbf{R r o t}_{M_{q}}$ is the range vector from radar to the real time location of the rotational point, $\mathbf{R}_{\mathrm{c}}$ is the range vector from radar to the center of wheel. Vector $\mathbf{R}_{\text {trans }}$ describes the translational motion of the center of wheel and vector $\widehat{\mathbf{r}}$ describes the rotational motion of point on the wheel. Moreover, $\left(x_{P_{c}}, y_{P_{c}}, z_{P_{c}}\right)$ is the initial coordinate of the wheel center, $R_{\mathrm{r}}$ is the distance between the scattering point and the center of wheel, $\omega$ is the angular velocity of the wheel and $\varphi$ is the initial phase angle of $M_{q}$.

For the scattering point $L_{\mathrm{m}}$ on the caterpillar band, when the point is on the upper part of caterpillar band, the instantaneous distance between the radar and $L_{\mathrm{m}}$ is

$$
\begin{aligned}
R_{L_{m}}(t) & =\left\|\operatorname{Reap}_{L_{m}}\right\|=\left\|\mathbf{Q} \mathbf{L}_{\mathbf{m}}+\mathbf{L}_{\mathbf{m}} \mathbf{L}_{\mathbf{m}}^{\prime}\right\|=f\left(x_{L_{m}}, y_{L_{m}}, z_{L_{m}}, \mathbf{v}, t\right) \\
& =\sqrt{\left(x_{L_{m}}+2 v t\right)^{2}+\left(y_{L_{m}}-H\right)^{2}+\left(z_{L_{m}}-R_{0}\right)^{2}}
\end{aligned}
$$

where $\mathbf{R c a p}_{L_{m}}$ is the range vector from radar to the real time location of the caterpillar band point, $\mathbf{Q} \mathbf{L}_{\mathbf{m}}$ is the vector from radar to the initial location of the point and vector $\mathbf{L}_{\mathbf{m}} \mathbf{L}_{\mathbf{m}}^{\prime}$ describes the motion of the point. When it moves to the lower part of caterpillar band, the instantaneous distance becomes the same as that of stationary point.

\subsection{Micro-Doppler Effect Analysis}

The transmitted signal of radar is a sinusoidal waveform expressed as [22]

$$
p(t)=\operatorname{rect}\left[\frac{t}{T_{\mathrm{p}}}\right] \exp \left(\mathrm{j} 2 \pi f_{\mathrm{c}} t\right)
$$

where $f_{\mathrm{c}}$ is the carrier frequency, $\operatorname{rect}(\cdot)$ means rectangular pulse envelope and $T_{\mathrm{p}}$ is the length of signal.

Assuming that numbers of stationary points, translational points, rotational points and caterpillar band points are $\left\{N_{\mathrm{tol}}, K_{\mathrm{tol}}, M_{\mathrm{tol}}, L_{\mathrm{tol}}\right\}$, the echo signal from the whole scene can be expressed as

$$
s(t)=\sum_{p=1}^{N_{\text {tol }}} s_{N_{p}}^{R}(t)+\sum_{n=1}^{K_{\text {tol }}} s_{K_{n}}^{R}(t)+\sum_{q=1}^{M_{\text {tol }}} s_{M_{q}}^{R}(t)+\sum_{m=1}^{L_{\text {tol }}} s_{L_{m}}^{R}(t)
$$

where $\mathbf{U}=\left\{N_{p}, K_{n}, M_{q}, L_{m}\right\}$ represents the kinds of scattering points. The returned signal of a single scattering point can be described as

$$
\begin{aligned}
& s_{P K}^{R}(t)=p\left(t-\frac{2 R_{\mathbf{U}}(t)}{c}\right) \\
& =\operatorname{rect}\left[\left(t-2 R_{\mathbf{U}}(t) / c\right) / T_{\mathrm{p}}\right] \cdot \exp \left[\mathrm{j} 2 \pi f_{\mathrm{c}}\left(t-2 R_{\mathbf{U}}(t) / c\right)\right]
\end{aligned}
$$

where $R_{\mathrm{U}}$ is the distance between radar and the scattering point. Through baseband transformation and time-frequency analysis, the micro-Doppler frequency can be calculated by

$$
f_{d-P K}=\frac{1}{2 \pi} \frac{\mathrm{d} \Phi_{\mathbf{U}}(t)}{\mathrm{d} t}=-\frac{2 f_{\mathrm{c}}}{c} \frac{\mathrm{d} R_{\mathbf{U}}(t)}{\mathrm{d} t}
$$


where $\Phi_{\mathbf{U}}(t)=2 \pi f_{\mathrm{c}}\left(t-2 R_{\mathbf{U}}(t) / c\right)$ is the phase of (7).

Assuming that far field assumption is satisfied $\left(R_{0}\right.$ is big enough), the instantaneous distances between scattering points and the radar can be approximated by Taylor expansion and $v^{2} t^{2} / 2 R_{0}$ can be subtracted from the distances of four kinds of scattering point by translational motion compensation. Then based on (8), the Doppler or micro-Doppler frequencies of four kinds of points can be obtained approximately by

1) stationary point:

$$
f_{d-N_{p}}(t)=-\frac{2 f_{\mathrm{c}}}{c} \frac{v^{2}}{z_{N_{p}}-R_{0}} t
$$

2) translational point:

$$
f_{d-K_{n}}(t)=-\frac{2 f_{\mathrm{c}}}{c} \frac{v x_{K_{n}}}{z_{K_{n}}-R_{0}},
$$

3) upper caterpillar band point:

$$
f_{d-L_{m}}(t)=\frac{2 f_{\mathrm{c}}}{c}\left(\frac{3 v^{2}}{z_{L_{m}}-R_{0}} t-\frac{2 v x_{L_{m}}}{z_{L_{m}}-R_{0}}\right),
$$

lower caterpillar band point:

$$
f_{d-L_{m}}(t)=f_{d-N_{p}}(t)
$$

4) rotational point:

$$
\begin{aligned}
& f_{d-M_{q}}(t)=-\frac{2 f_{c}}{c}\left\{-\frac{v x_{p c}}{z_{p c}-R_{0}}+\frac{R_{r}}{z_{p c}-R_{0}}\left[\left(\omega y_{p c}-\omega H+v\right)^{2}\right.\right. \\
& \left.\left.+\left(\omega\left(x_{p c}+v t\right)\right)^{2}\right]^{1 / 2} \sin (\theta+\omega t+\varphi)\right\}
\end{aligned}
$$

where

$$
\begin{gathered}
\varphi=\arctan \left(\frac{y_{M_{q}}-y_{c}}{x_{M_{q}}-x_{c}}\right), \\
\theta=\arctan \left[\frac{\omega\left(x_{p c}+v t\right)}{\omega y_{p c}-\omega H+v}\right] .
\end{gathered}
$$

The micro-Doppler frequency of rotational point is a sinusoidal curve, whose frequency is

$$
\begin{aligned}
f_{f_{d-M_{q}(t)}} & =\frac{\mathrm{d}}{\mathrm{d} t}(\theta+\omega t+\varphi) \\
& =\omega+\frac{\omega v\left(\omega y_{p c}-\omega H+v\right)}{\left(\omega y_{p c}-\omega H+v\right)^{2}+\left[\omega\left(x_{p c}+v t\right)\right]^{2}} \approx \omega .
\end{aligned}
$$

As can be seen from (9) to (13), the Doppler frequency curves of stationary scattering points on the timefrequency spectrogram are straight lines with negative slopes. On the contrary, the curves of upper points on the caterpillar band are straight lines with positive slopes. The translational points correspond to lines with zero slopes.
The curves of rotational points are sinusoids with different amplitudes and phases. Therefore, by extracting the features of the sinusoids, physical parameters of wheels can be acquired.

\section{MP-Based Wheel Parameters Extraction}

In order to obtain accurate rotational features, the echo signals generated by stationary points, translational points and upper caterpillar band points, namely irrelevant signals, need to be removed first.

Sparse representation uses a linear combination of basic signals selected from the dictionary to reconstruct the original signal. MP is a typical sparse decomposition method. The signal can be decomposed into an expansion form of functions in the non-orthogonal basis by MP. Once the returned signal is sparsely represented, the removal of irrelevant signal and extraction of features will become much easier.

\subsection{Dot Matrix Description of a Scene}

The radar echo of a moving vehicle in the scene is mainly generated by the scattering points discussed in Sec. 2, and then the components of the echo signal can be reconstructed according to the motion states of scattering points.

For the simulation validation, a model of a moving tank with caterpillar tracks on the ground is built by Google Sketchup to illustrate the method, which is shown in Fig. 3. The scene is described with a $3 \mathrm{D}$ dot matrix which is big enough to cover all the scattering points on the target. In order to guarantee the simulation accuracy, the interval of points in the $3 \mathrm{D}$ matrix must be smaller than the radar resolution.

For the translational points on the vehicle, the $3 \mathrm{D}$ dot matrix is represented by $C_{\text {trans }}$ and $\operatorname{card}\left(C_{\text {trans }}\right)=K$ is the number of the dots. For a $\operatorname{dot}\left(x t_{k}, y t_{k}, z t_{k}\right) \in C_{\text {trans }}$, calculate the distance between the dot and radar according to (2) and build an analogous radar signal $a_{k}(t)$, which is expressed as

$$
a_{k}(t)=\exp \left[-\mathrm{j} 4 \pi f_{\mathrm{c}} \frac{\sqrt{\left(x t_{k}+v t\right)^{2}+\left(y t_{k}-H\right)^{2}+\left(z t_{k}-R_{0}\right)^{2}}}{c}\right]
$$

The atom $a_{k}(t)$ is considered as the radar echo signal of the translational point after baseband transformation. The radar signals of all the translational points construct a signal set $\Pi_{\text {trans }}=\left\{a_{k}(t) \mid a_{1}(t), a_{2}(t), \ldots, a_{K}(t)\right\}$, which is considered as the signal dictionary of translational points.

In a similar way, the signals of stationary points exist on the ground and the lower part of caterpillar band can be given by $\Pi_{\text {sta }}=\left\{b_{n}(t) \mid b_{1}(t), b_{2}(t), \ldots, b_{N}(t)\right\}$. The dictionary of 


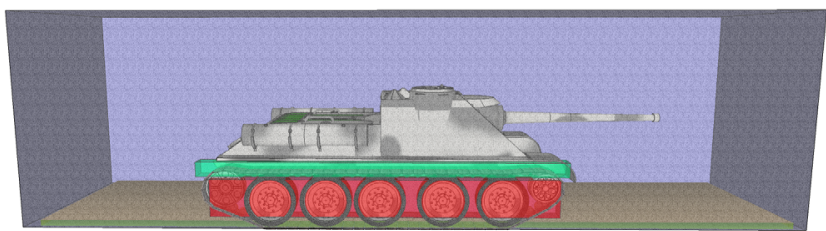

Fig. 3. A dot matrix description of a scene.

the upper caterpillar band points shown as the green area in Fig. 3 can be represented as $\Pi_{\text {cap }}=\left\{c_{l}(t) \mid c_{1}(t), c_{2}(t), \ldots, c_{L}(t)\right\}$. For stationary point $\left(x s_{n}, y s_{n}, z s_{n}\right)$ and the upper caterpillar band point $\left(x p_{l}, y p_{l}, z p_{l}\right), b_{n}(t)$ and $c_{l}(t)$ can be expressed by

$$
\begin{aligned}
& b_{n}(t)=\exp \left[-\mathrm{j} 4 \pi f_{\mathrm{c}} \frac{\sqrt{x s_{n}^{2}+\left(y s_{n}-H\right)^{2}+\left(z s_{n}-R_{0}\right)^{2}}}{c}\right],(18) \\
& c_{l}(t)=\exp \left[-\mathrm{j} 4 \pi f_{\mathrm{c}} \frac{\sqrt{\left(x p_{l}+2 v t\right)^{2}+\left(y p_{l}-H\right)^{2}+\left(z p_{l}-R_{0}\right)^{2}}}{c}\right]
\end{aligned}
$$

where $\left(x s_{n}, y s_{n}, z s_{n}\right)$ and $\left(x p_{l}, y p_{l}, z p_{l}\right)$ are the coordinates of a stationary point and upper caterpillar band point in the 3D matrixes, respectively.

\subsection{Removal of Irrelevant Signals}

Take the translational points as example to give a description of irrelevant signals removal based on the ideas of MP. Assuming that a received radar echo signal is $s(t)$, calculate the correlation coefficient $d_{k}$ between $s(t)$ and $a_{k}(t)$. All the correlation coefficients $\left\{d_{k}\right\}$ between $s(t)$ and every elements in $\Pi_{\text {trans }}$ can be given by

$$
\left\{d_{k} \mid d_{k}=\left\langle s(t), a_{k}(t)\right\rangle ; a_{k}(t) \in \Pi_{\text {trans }}\right\}
$$

where $\langle\cdot\rangle$ is the inner product.

Then, search the maximum value $d_{i}$ in $\left\{d_{k}\right\}$ and record the corresponding sequence number $i$. Thus, $a_{i}(t)$ becomes the most relevant signal component generated by the translational scattering points, which needs to be removed. The echo signal is updated by

$$
s^{\prime}(t)=s(t)-d_{i} \cdot \frac{a_{i}(t)}{\left\|a_{i}(t)\right\|} .
$$

Exclude the element $a_{i}(t)$ from $\Pi_{\text {trans }}$ and calculate the correlation coefficients between $s^{\prime}(t)$ and $\Pi_{\text {trans }}$ again. Conduct the iterative processing to remove the most relevant signal component until the maximum correlation coefficient is smaller than the threshold value. At this point, the signal components induced by the translational scattering points have been mostly removed.

Based on the atom sets $\Pi_{\text {sta }}$ and $\Pi_{\text {cap }}$ of stationary points and upper caterpillar band, the corresponding signal components can also be removed from the radar echoes by performing the same steps.

\subsection{Extraction of Wheel Parameters}

After the removal of irrelevant signal, the micro-Doppler signals generated by the rotational wheels become the major components of the echo signal. All the scattering points on the wheels move in a circular motion, generating modulated sinusoidal signals with different amplitudes and phases. In this case, the parameters of wheels can be extracted based on MP and the micro-Doppler features of rotational points.

The red zone in Fig. 3 is the $3 \mathrm{D}$ dot matrix to cover the wheels, expressed as $C_{\mathrm{r}}$. The atom set of each dot in the matrix is formulated according to the signals generated by the scattering points around the centers of wheels. Based on MP, the most relevant atom of each dot with the echo signal can be acquired. If the correlation coefficient is big enough, the dot might be the center of wheel. In order to estimate the parameters of wheels more accurately, each atom set is generated by eight points around the dot in the matrix, shown as blue points in Fig. 4. The eight points are expressed as a set $\Pi_{w p}$ and $d_{x}$ is the interval which is smaller than the radar resolution.

The red point is a dot in the 3D matrix, which might be the center of wheel. Assuming that the frequencies of wheels are within the range of $\left(\omega_{\min }, \omega_{\max }\right)$, the atom set of each blue point can be expressed as

$$
\Pi_{\text {rot }}\left(x_{a}, y_{a}, z_{a}\right)=\left\{o_{m}(t) \mid o_{1}(t), o_{2}(t), \cdots, o_{\omega_{\max }-\omega_{\min }+1}(t)\right\}
$$

where

$$
o_{m}(t)=\exp \left[-\mathrm{j} 4 \pi \frac{f_{\mathrm{c}} R_{\mathrm{r}}(t)}{c}\right],
$$

$$
\begin{aligned}
R_{\mathrm{r}}(t) & =\left[\left(x r_{m}+v t\right)^{2}+\left(y r_{m}-H\right)^{2}+R_{r}^{2}+\left(z r_{m}-R_{0}\right)^{2}\right. \\
& \left.+2 R_{r} \sqrt{\left(x r_{m}-v t\right)^{2}+\left(y r_{m}-H\right)^{2}} \sin (\theta+\omega t+\varphi)\right]^{\frac{1}{2}}
\end{aligned}
$$

where $\quad\left(x r_{m}, y r_{m}, z r_{m}\right) \in C_{\mathrm{r}}, \quad\left(x_{a}, y_{a}, z_{a}\right) \in \Pi_{w p}$, $\omega \in\left(\omega_{\min }, \omega_{\max }\right), \quad R_{r}=\sqrt{2} d_{x}, \theta=\arctan \left[\left(x r_{m}+v t\right) / y r_{m}\right]$, and $\varphi=\arctan \left[\left(y_{a}-y r_{m}\right) /\left(x_{a}-x r_{m}\right)\right]$.

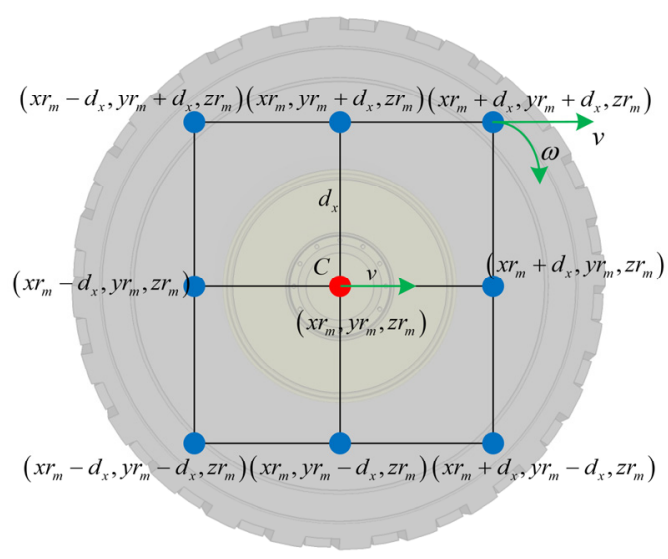

Fig. 4. Points for rotational atom set generation. 
Each blue point in Fig. 4 will generate a signal set with $\left(\omega_{\max }-\omega_{\min }+1\right)$ atoms, and thus the number of atoms in the set of each dot in the 3D matrix is $\left(\omega_{\max }-\omega_{\min }+1\right) \times 8$. The correlation coefficients of eight points are calculated separately by

$$
\left\{d_{m} \mid d_{m}=\left\langle s_{r}(t), o_{m}(t)\right\rangle ; o_{m}(t) \in \Pi_{\mathrm{rot}}\left(x_{a}, y_{a}, z_{a}\right)\right\}
$$

where $s_{r}(t)$ is the signal after the removal of irrelevant signals. Each calculation in connection with each blue point will generate a maximum correlation coefficient, and a frequency value when the correlation coefficient reaches the maximum. In other words, in the calculations of each dot in the $3 \mathrm{D}$ matrix, eight maximum correlation coefficients $\left\{D_{1}, D_{2}, \ldots, D_{8}\right\}$ and the corresponding frequencies $\omega_{r}=\left\{\omega_{1}, \omega_{2}, \ldots, \omega_{8}\right\} \in\left(\omega_{\min }, \omega_{\max }\right)$ will be obtained.

In the ideal state, the frequencies $\left\{\omega_{1}, \omega_{2}, \ldots, \omega_{8}\right\}$ will be all equal to the rotational frequency if the current red dot is the center of wheel. Nevertheless, the modulation signals of rotational scattering points are different, which will cause interferences on each other. Hence, the eight extraction results of frequency might be different. If the eight frequencies are all different, the current red dot may not be the center of wheel. On the contrary, if there are same elements in $\omega_{r}$, the red dot at this moment might be the center of wheel. The frequency of them, denoted as $\omega_{t}$, is the estimated rotational frequency of the wheel. Providing that the serial numbers of the repeated elements are $W=\left\{r \mid \omega_{r}=\omega_{t}\right\}$, the output correlation coefficients and frequencies are determined as follows:

$$
\begin{aligned}
& \text { IF } \quad \operatorname{card}(W) \geq 3 \\
& \boldsymbol{\omega}\left(x_{r m}, y_{r m}, z_{r m}\right)=\omega_{t} \\
& \mathbf{D}\left(x_{r m}, y_{r m}, z_{r m}\right)=\max \left\{D_{r}\right\} \\
& \mathrm{ELSE} \\
& \boldsymbol{\omega}\left(x_{r m}, y_{r m}, z_{r m}\right)=\omega_{1} \\
& \mathbf{D}\left(x_{r m}, y_{r m}, z_{r m}\right)=\min \left\{D_{1}, D_{2}, \cdots, D_{8}\right\}
\end{aligned}
$$

After the calculation of all points in $C_{\mathrm{r}}$, matrices of correlation coefficients and frequencies shown in (26) can be obtained. If a certain point in $C_{\mathrm{r}}$ is the center of a vehicle's wheel, the correlation coefficient on that coordinates will be much bigger than other values. Therefore, the local maxima in the correlation coefficient matrix $\mathbf{D}$ shows the estimated coordinates of wheels. Once the coordinates of wheels are obtained, the estimated rotational frequencies can also be obtained from $\boldsymbol{\omega}$. The radiuses of wheels $R$ can be calculated by the frequencies $\omega$ and the velocity of target $v: R=v / \omega$.

\subsection{Procedure of MP-Based Vehicle Parameters Extraction}

Based on the principle of MP, a wheel parameter extraction method of a moving vehicle through micro-Doppler frequencies is proposed in this section. The simulation indicates that the signal components of other scattering points in addition to the rotational ones will be detrimental to the extraction of micro-Doppler frequency. Hence, on the basis of micro-Doppler signal form, three signal atom sets are built to suppress those irrelevant signals. Then the fourth atom set is formed according to the motion state of rotational scattering points, which is used to pursuit those sinusoids with different amplitudes and phases and extract the features of wheels further. The general framework of algorithm is shown in Fig. 5.

The process of the proposed method can be summarized as Algorithm 1.

\begin{tabular}{l}
\hline Algorithm 1: Wheel parameters extraction via micro- \\
Doppler feature based on matching pursuit \\
\hline Input: the radar echo of target $s(t)$, the translational \\
points set $\Pi_{\text {trans, the stationary points set } \Pi_{\text {sta, the }}}$ \\
caterpillar band points set $\Pi_{\text {cap }}$, the rotational points set \\
$\Pi_{\text {rot }}$ the threshold value $T$. \\
Initialization: \\
do \\
1. Calculate the correlation coefficients between $s(t)$ \\
and $\Pi_{\text {trans }}$ with $(20)$. \\
2. Exclude the element $a_{i}$ from $\Pi_{\text {trans }}$. \\
3. Update the echo signal with $(21)$. \\
while $a_{i}>T$ \\
4. Change $\Pi_{\text {trans }}$ into $\Pi_{\text {sta }}, \Pi_{\text {cap }}$ and repeat steps $1 \sim 3$. \\
5. Calculate the correlation coefficients between the \\
residual signal and $\Pi_{\text {rot }}$ with $(25)$. \\
6. Output the elements in correlation coefficients matrix \\
and frequency matrix with $(26)$. \\
Output: Correlation coefficients matrix and frequency \\
matrix.
\end{tabular}

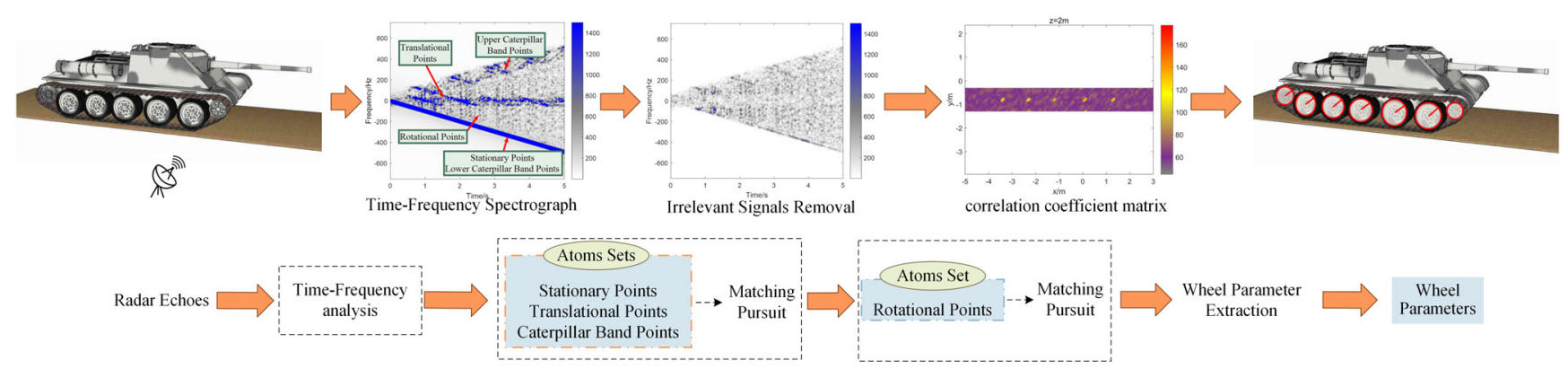

Fig. 5. General framework of the proposed vehicle parameter extraction method. 


\section{Simulations and Discussion}

In this section, some simulation experiments are performed to validate the proposed method. The programs are written via Microsoft Visual Studio 2012. All the analyses and programs are performed on a Win10 operated workstation, whose CPU is Inter Core $19-9900 \mathrm{~K}$ and RAM is $16 \mathrm{G}$.

Firstly, geometry models of armored car and tank with caterpillar band are built to test the wheel parameters extraction under the noiseless condition. The radar transmits a sinusoidal waveform and the detailed parameters of radar are listed in Tab. 1. For the signal after baseband transformation, the sampling rate of $1500 \mathrm{~Hz}$ satisfies Nyquist sampling theorem.

\subsection{Wheels Parameters Extraction of Moving Caterpillar Tank}

In order to test the effectiveness of the algorithm for caterpillar tank with wheels of different sizes, a model of a moving caterpillar tank on the ground is built, shown in Fig. 1. The geometrical relationship between the radar and target is shown in Fig. 2. The target is moving along the $\mathrm{x}$-axis in the positive direction at the speed of $10 \mathrm{~m} / \mathrm{s}$, whose parameters of wheels are listed in Tab. 2 .

\begin{tabular}{|c|c|c|c|}
\hline Parameter & Value & Parameter & Value \\
\hline Carrier frequency & $30 \mathrm{GHz}$ & Radar coordinates & $(0,1.7,200) \mathrm{m}$ \\
\hline Sampling frequency & $1500 \mathrm{~Hz}$ & Dwell time & $5 \mathrm{~s}$ \\
\hline
\end{tabular}

Tab. 1. Radar parameters.

\begin{tabular}{|c|c|c|}
\hline Serial number & $\begin{array}{c}\text { Initial coordinates of } \\
\text { centers/m }\end{array}$ & Radius/cm \\
\hline 1 & $(-3.4,-0.8,2.0)$ & 54 \\
\hline 2 & $(-2.3,-0.8,2.0)$ & 54 \\
\hline 3 & $(-1.2,-0.8,2.0)$ & 54 \\
\hline 4 & $(0.1,-0.8,2.0)$ & 54 \\
\hline 5 & $(1.3,-0.8,2.0)$ & 54 \\
\hline 6 & $(-4.4,-0.6,1.9)$ & 31 \\
\hline 7 & $(2.3,-0.5,1.9)$ & 38 \\
\hline
\end{tabular}

Tab. 2. Wheel parameters of tank.

Echo signals are simulated according to (5), (6) and Short Time Fourier Transform (STFT) is used to derive the time-frequency (TF) spectrogram. The original TF spectrogram is obtained by applying STFT to the echo signal, shown in Fig. 6. After motion compensation, the scattering points on the ground and lower caterpillar band, namely stationary points, form many lines at the bottom of the curves with negative slopes. The slopes of the lines formed by translational scattering points on the body of the car are zero.

Many sinusoids are formed by the rotational scattering points on the wheels, whose amplitudes and phases are different. All the results are consistent with (9)-(13). As

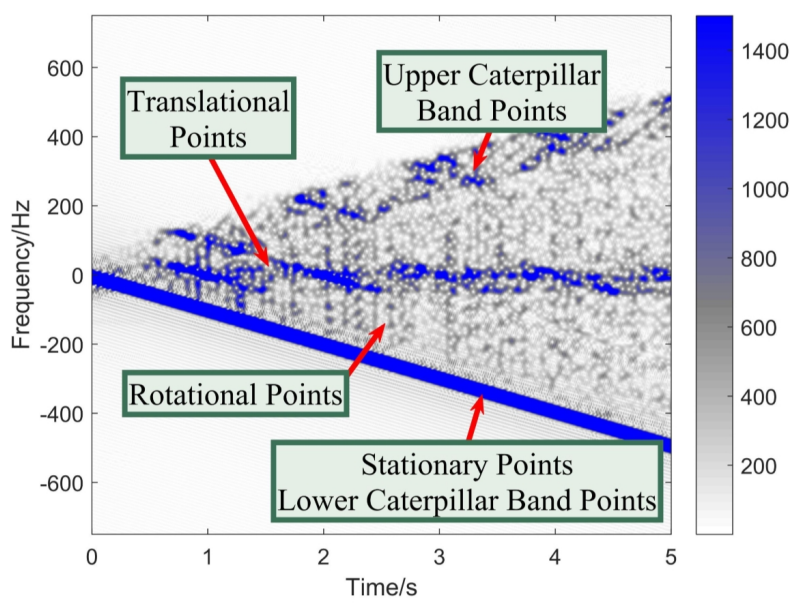

Fig. 6. Original TF spectrogram of the caterpillar tank scene.

shown in the spectrogram, the signal components generated by stationary points and translational points are much stronger than the micro-Doppler signals generated by rotational points. Moreover, the signal components of upper caterpillar band points also cause interference on the micro-Doppler signals. Thus, if we want to extract the parameters of wheels accurately, the removal of irrelevant signals is quite necessary.

The original scattering points in the scene distribute at the range of ( -7.7 to $7.8,-1.5$ to $1.4,-2.3$ to 2.2$) \mathrm{m}$ with $0.1 \mathrm{~m}$ interval along the $\mathrm{x}-, \mathrm{y}-$, and $\mathrm{z}$-axes. During the process of irrelevant signal removal, the coordinates of the points in the dot matrix corresponding to the translational points are set to (-5 to $5,-1.8$ to $1.8,-2.5$ to 2.5 ) $\mathrm{m}$ with $0.1 \mathrm{~m}$ intervals and those corresponding to the stationary points are set to ( -8 to $8,-1.8$ to $-1.3,-2.5$ to 2.5 ) $\mathrm{m}$ with the same intervals. The coverage of those points is small in order to cut down the amount of computation. But the actual situation is that we do not know the precise coordinates of the target, so the coverage of the points can be bigger.

The spectrogram after irrelevant signal removal is shown in Fig. 7. Obviously, the irrelevant signal components have been removed a lot and signals generated by rotational points become the major components of the echo.

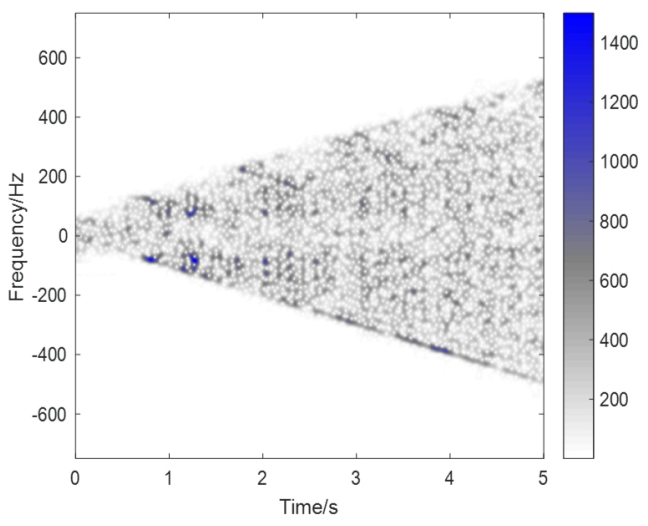

Fig. 7. TF spectrogram after irrelevant signal removal of the caterpillar tank scene. 


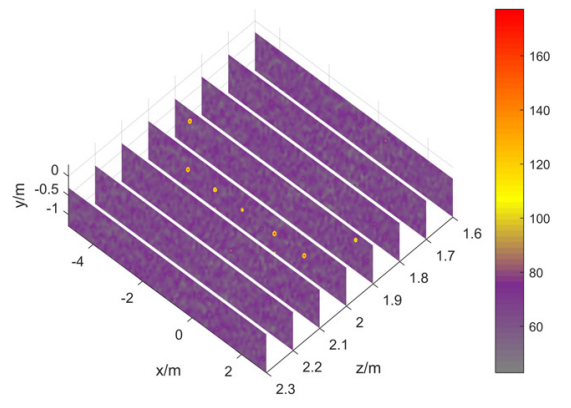

(a)

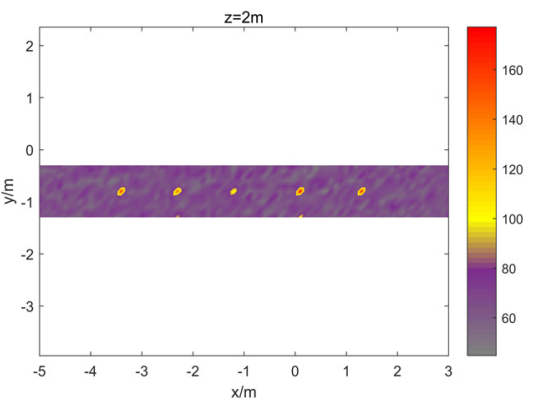

(b)

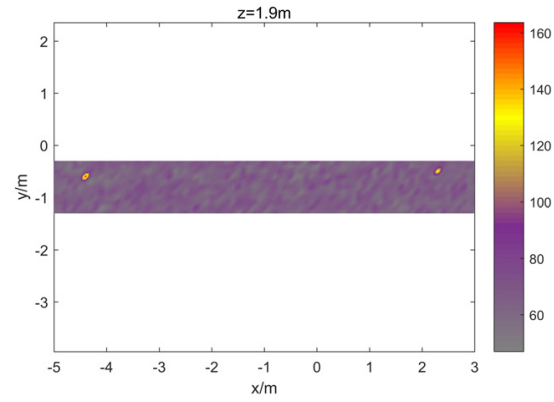

(c)

Fig. 8. Wheels parameters extraction of tank model. (a) Correlation coefficient of the whole $3 \mathrm{D}$ dot matrix. (b) Correlation coefficient when $z=2 \mathrm{~m}$. (c) Correlation coefficient when $z=1.9 \mathrm{~m}$.

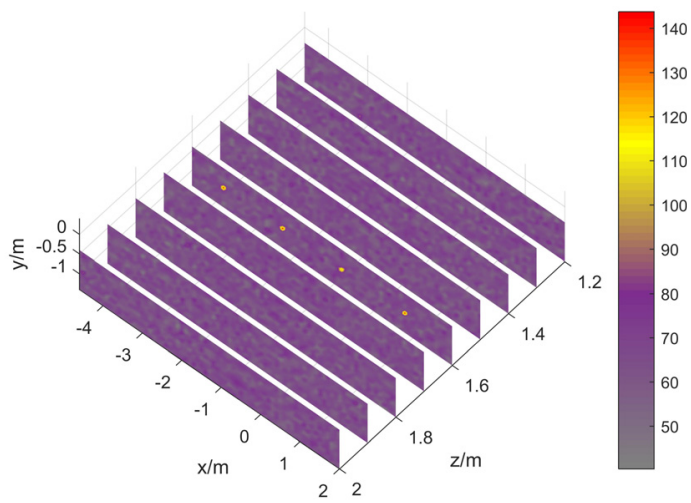

(a)

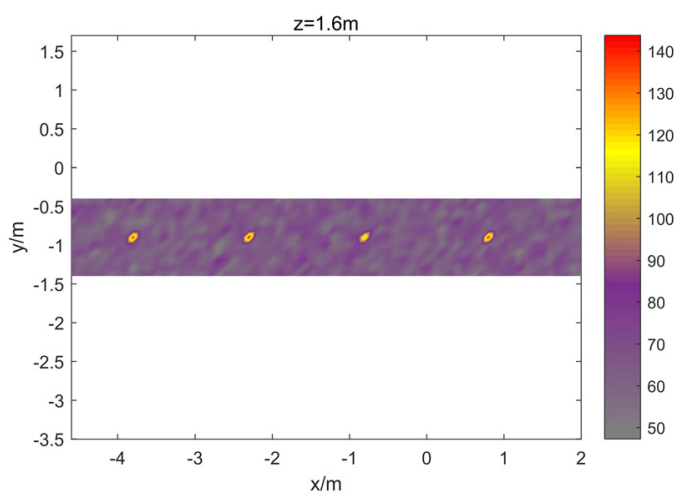

(b)

Fig. 9. Wheel parameters extraction of armored car. (a) Correlation coefficient of the whole 3D dot matrix. (b) Correlation coefficient when $z=1.6 \mathrm{~m}$.

Then the dot matrix $\boldsymbol{C}_{\mathrm{r}}$ is created to cover the centers of all wheels, whose coverage is also small to cut down the amount of computation. The coordinates of the dots in the matrix are (-5 to $3,-1.3$ to $0.3,1.6$ to 2.3$) \mathrm{m}$ at $0.1 \mathrm{~m}$ intervals.

The correlation coefficient matrix is shown in Fig. 8(a). Each point in the matrix $\mathbf{C}_{\mathrm{r}}$ will generate a correlation coefficient and its value is corresponding to the color depth refer to the color bar. Apparently, seven local maxima can be found when $z=1.9 \mathrm{~m}$ and $2.0 \mathrm{~m}$, which means there are seven wheels on the side that the radar illuminates on. The coordinates of those maxima can also be acquired, which are identified as the initial locations of wheels.

The frequencies of wheels can be obtained by searching the frequency matrix $\omega$ according to the coordinates of the maxima. The frequencies of wheels $1-5$ are $18.5 \mathrm{rad} / \mathrm{s}$, wheel 6 is $32.3 \mathrm{rad} / \mathrm{s}$ and wheel 7 is $26.3 \mathrm{rad} / \mathrm{s}$. Radiuses can be calculated after the conversion, which are equal to those listed in Tab. 2.

\subsection{Wheels Parameters Extraction of Moving Armored Car}

A similar experiment of armored car is performed in our previous work [23]. In this paper, the armored car

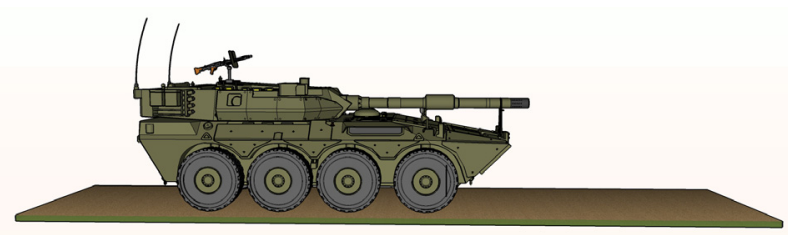

Fig. 10. Armored car on the ground.

\begin{tabular}{|c|c|c|}
\hline Serial number & $\begin{array}{c}\text { Initial coordinates of } \\
\text { centers/m }\end{array}$ & Radius/cm \\
\hline 1 & $(-3.8,-0.9,1.6)$ & 60 \\
\hline 2 & $(-2.3,-0.9,1.6)$. & 60 \\
\hline 3 & $(-0.8,-0.9,1.6)$ & 60 \\
\hline 4 & $(0.8,-0.9,1.6)$ & 60 \\
\hline
\end{tabular}

Tab. 3. Wheel parameters of armored car.

model, shown in Fig. 10, is used again to prove that better extraction results can be obtained by improving the decision rule of the output estimated parameters. There are four wheels on one side of the vehicle and the detailed information is shown in Tab. 3.

The same procedures are conducted to remove the irrelevant signals. The correlation coefficient matrix is shown in Fig. 9(a). Obviously, four wheels can be detected and the coordinates of them are consistent with the actual 
parameters. The frequencies of wheels are all $16.7 \mathrm{rad} / \mathrm{s}$. Thus, the radiuses can be calculated to be $60 \mathrm{~cm}$ according to the velocity of vehicle.

Comparing to the extraction results in our previous work [23], the locations of wheels in the correlation coefficient matrix are much clearer. The maxima that may cause interference are almost eliminated, which makes the extraction of wheels' numbers and positions more accurate.

\subsection{Performance Evaluation under Various SNRs}

To test the anti-noise capability of the algorithm, Gaussian white noises under different SNRs are added to the echo signal of armored car. To reduce the amount of calculation, only the position and radius of the first wheel of armored car are extracted. The radius of the wheel is $60 \mathrm{~cm}$ and the initial coordinate of it is $(-3.8,-0.9,1.6) \mathrm{m}$. The SNRs are set up from $-10 \mathrm{~dB}$ to $5 \mathrm{~dB}$ with the interval of $0.5 \mathrm{~dB}$.

The estimated position error and radius are tested under different SNRs. The position error is the distance between the estimated center of the wheel and the actual center. At each SNR, the radius of the wheel is estimated with 50 trials. The SNR of noise per trial is the same but the values are different. The position error and the estimated radius are averaged at each SNR to obtain the results, which are shown in Fig. 11.

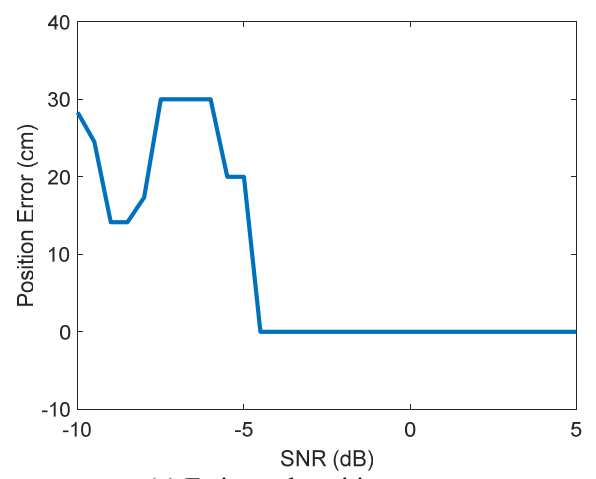

(a) Estimated position error

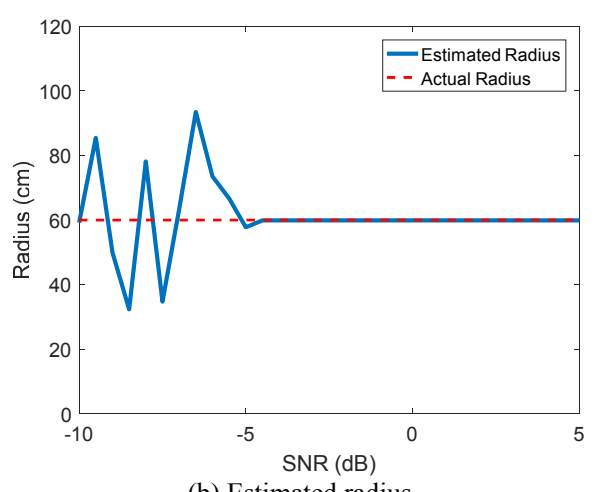

(b) Estimated radius

Fig. 11. Estimated results under different SNRs.
As can be seen from the results, when the SNR is lower than $-5 \mathrm{~dB}$, the maximum estimated position error of the wheel's center is about $30 \mathrm{~cm}$. Besides, the estimated radius is not quite accurate compared to the real value of radius. As the SNR increases to $-5 \mathrm{~dB}$, the estimated results of position and radius of the wheel can be obtained with acceptable accuracy.

\section{Conclusion}

A vehicle wheel parameters extraction method based on micro-Doppler features and MP is proposed in this paper. The micro-Doppler effects of stationary points, translational points and the caterpillar band points on the vehicle are analyzed. Simulation results validate that the proposed method can accurately extract the wheel parameters, including the numbers, positions and radiuses of wheels. The algorithm has been proved to be effective for vehicles with caterpillar band. Moreover, this algorithm has acceptable anti-noise performance. Future work will use the realistic measured signal to validate the proposed method and the effect of the tracking distances on estimation accuracy will be analyzed.

\section{Acknowledgments}

This work was supported in part by the National Natural Science Foundation of China under Grant 61871158 , and in part by the Scientific Research Foundation for the Returned Overseas Scholars of Heilongjiang Province under Grant LC2018029.

\section{References}

[1] CHEN, V. C., LI, F., HO, S. et al. Micro-Doppler effect in radar: Phenomenon, model, and simulation study. IEEE Transactions on Aerospace and Electronic Systems, 2006, vol. 42, no. 1, p. 2-21. DOI: 10.1109/TAES.2006.1603402

[2] TANG, B. Micro-Doppler effect of extended streamlined targets based on sliding scattering center mode. Radioengineering, 2016, vol. 25, no. 2, p. 68-74. DOI: 10.13164/re.2016.0268

[3] ZHU, L., ZHANG, S., ZHAO, H., et al. Classification of UAV-toground vehicles based on micro-Doppler signatures using singular value decomposition and deep convolutional neural networks. IEEE Access, 2019, vol. 7, p. 22133-22143. DOI: 10.1109/ACCESS.2019.2898642

[4] JIAN, M., LU, Z., CHEN, V. C. Experimental study on radar micro-Doppler signatures of unmanned aerial vehicles. In 2017 IEEE Radar Conference (RadarConf). Seattle (WA, USA), 2017, p. 854-857. DOI: 10.1109/RADAR.2017.7944322

[5] SINGH, A. K., KIM, Y. Automatic measurement of blade length and rotation rate of drone using W-band micro-Doppler radar. IEEE Sensors Journal, 2018, vol. 18, no. 5, p. 1895-1902. DOI: 10.1109/JSEN.2017.2785335 
[6] CAO, P., XIA, W., Y, LI. Classification of ground targets based on radar micro-Doppler signatures using deep learning and conventional supervised learning methods. Radioengineering, 2018 , vol. 27, no. 3, p. 835-845. DOI: 10.13164/RE.2018.0835

[7] ZHAO, Y., SU, Y. The extraction of micro-Doppler signal with EMD algorithm for radar-based small UAVs' detection. IEEE Transactions on Instrumentation and Measurement, 2020, vol. 69, no. 3, p. 929-940. DOI: 10.1109/TIM.2019.2905751

[8] CHEN, V. C., LING, H. Time-Frequency Transforms for Radar Imaging and Signal Analysis. Norwood (USA): Artech House, 2002. ISBN: 978-1580532884

[9] AUGER, F., FLANDRIN, P. Improving the readability of timefrequency and time-scale representations by the reassignment method. IEEE Transactions on Signal Processing, 1995, vol. 43, no. 5, p. 1068-1089. DOI: 10.1109/78.382394

[10] GU, F., FU, M., LIANG, B., et al. Translational motion compensation and micro-Doppler feature extraction of space spinning targets. IEEE Geoscience and Remote Sensing Letters, 2018, vol. 15, no. 10, p. 1550-1554. DOI: 10.1109/LGRS.2018.2849869

[11] CAI, C., LIU, W., FU, J., et al. Empirical mode decomposition of micro-Doppler signature. In IEEE International Radar Conference. Arlington (VA, USA), 2005, p. 895-899. DOI: 10.1109/RADAR.2005.1435954

[12] LI, G., VARSHNEY, P. K. Micro-Doppler parameter estimation via parametric sparse representation and pruned orthogonal matching pursuit. IEEE Journal of Selected Topics in Applied Earth Observations and Remote Sensing, 2014, vol. 7, no. 12, p. 4937-4948. DOI: 10.1109/JSTARS.2014.2318596

[13] MALLAT, S. G., ZHANG, Z. Matching pursuits with timefrequency dictionaries. IEEE Transactions on Signal Processing, 1993, vol. 41, no. 12, p. 3397-3415. DOI: 10.1109/78.258082

[14] LUO, Y., ZHANG, Q., QIU, C., et al. Micro-Doppler feature extraction for wideband imaging radar based on complex image orthogonal matching pursuit decomposition. IET Radar Sonar \& Navigation, 2013, vol. 7, no. 8, p. 914-924. DOI: 10.1049/ietrsn.2012.0327

[15] DU, L., LI, L., WANG, B., et al. Micro-Doppler feature extraction based on time-frequency spectrogram for ground moving targets classification with low-resolution radar. IEEE Sensors Journal, 2016, vol. 16, no. 10, p. 3756-3763. DOI: 10.1109/JSEN.2016.2538790

[16] ZHU, L., ZHANG, S., MA, et al. Classification of UAV-to-ground targets based on enhanced micro-Doppler features extracted via PCA and compressed sensing. IEEE Sensors Journal, 2020, vol. $20, \quad$ no. 23, p. 14360-14368. DOI: 10.1109/JSEN.2020.3008439

[17] DING, Y, SUN, Y., HUANG, G., et al. Human target localization using Doppler through-wall radar based on micro-Doppler frequency estimation. IEEE Sensors Journal, 2020, vol. 20, no. 15, p. 8778-8788, DOI: 10.1109/JSEN.2020.2983104

[18] YI, L., XIA, W., DONG, S. Time-based multi-component irregular FM micro-Doppler signals decomposition via STVMD. IET Radar, Sonar \& Navigation, 2020, vol. 14, no. 10, p. 1502-1511. DOI: 10.1049/iet-rsn.2020.0091

[19] ZHAI, S., MAO, T., HU, B. An EMD-based micro-Doppler signature for moving vehicles classification. In 2019 IEEE 5th International Conference on Computer and Communications (ICCC). Chengdu (China), 2019, p. 1322-1326. DOI: 10.1109/ICCC47050.2019.9064359

[20] OH, B., GUO, X., WAN, F., et al. Micro-Doppler mini-UAV classification using empirical-mode decomposition features. IEEE Geoscience and Remote Sensing Letters, 2018, vol. 15, no. 2, p. 227-231. DOI: 10.1109/LGRS.2017.2781711
[21] ZHU, L., ZHANG, S., XU, S., et al. Classification of UAV-toground targets based on micro-Doppler fractal features using IEEMD and GA-BP neural network. IEEE Sensors Journal, 2020, vol. 20 , no. 1 , p. 348-358. DOI: 10.1109/JSEN.2019.2942081

[22] CHEN, V. C., TAHMOUSH, D., MICELI, W. J. (Eds.) Radar Micro-Doppler Signatures: Processing and Applications. London (UK): The Institution of Engineering and Technology, 2014. ISBN: 9781849197168

[23] CHEN, Y., WANG, N., ZHANG, L. Moving vehicle wheel parameter extraction via micro-Doppler feature based on matching pursuit. In 2020 Cross Strait Radio Science \& Wireless Technology Conference (CSRSWTC). Fuzhou (China), 2020, p. 1-3. DOI: 10.1109/CSRSWTC50769.2020.9372705

\section{About the Authors ...}

Lamei ZHANG received the B.S., M.Sc., and Ph.D. degrees in Information and Communication Engineering from the Harbin Institute of Technology, Harbin, China, in 2004, 2006 and 2010, respectively. Currently, she is an Associate Professor with the Department of Information Engineering, Harbin Institute of Technology. She serves as the Secretary of the IEEE Harbin Geoscience and Remote Sensing (GRSS) Chapter. Her research interests include remote sensing images processing, information extraction, and intelligent interpretation of high-resolution SAR, polarimetric SAR, and polarimetric SAR interferometry.

Yifan CHEN was born in Wuxi, China in 1996. He received the B.S. degree from the School of Physics and Engineering, Zhengzhou University, Zhengzhou, China, in 2018. He is currently a Ph.D.'s degree student at the Department of Information Engineering, Harbin Institute of Technology, Harbin, China. His research interest is the scattering properties and characteristics of radar targets.

Shuo LIU was born in Harbin, China, in 1993. He received the B.Sc. degree from the Harbin Engineering University, Harbin, in 2016, and the M.Sc. degree from the Harbin Institute of Technology, Harbin, in 2018. He is currently pursuing the Ph.D. degree with the Department of Information Engineering, Harbin Institute of Technology, Harbin, China. He is currently working on the high-performance electromagnetic simulation and its application to remote sensing.

Bin ZOU (corresponding author) received the B.S. degree in Electronic Engineering from the Harbin Institute of Technology in 1990, M.Sc. degree of Space Studies from the International Space University, Strasbourg, France, in 1998, and Ph.D. degree in Information and Communication Engineering from the Harbin Institute of Technology, Harbin, China, in 2001. He is currently a Professor with the Department of Information Engineering, Harbin Institute of Technology. He serves as the chair of the IEEE Harbin Geoscience and Remote Sensing (GRSS) Chapter. His research interests include SAR image processing, polarimetric SAR, and polarimetric SAR interferometry. 